\title{
The nonlinear Kneser problem for singular in phase variables second-order differential equations
}

Nino Partsvania ${ }^{1,2^{*}}$ and Bedřich Pưža ${ }^{3}$

Dedicated to our dear teacher, Prof. Ivan Kiguradze

*Correspondence: ninopa@rmi.ge

${ }^{1}$ A. Razmadze Mathematical Institute of I. Javakhishvili Tbilisi

State University, 6 Tamarashvili St.,

Tbilisi, 0177, Georgia

${ }^{2}$ International Black Sea University, 2 David Agmashenebeli Alley 13km,

Tbilisi, 0131, Georgia

Full list of author information is

available at the end of the article

\section{Abstract}

For the singular in phase variables differential equation

$$
u^{\prime \prime}=f\left(t, u, u^{\prime}\right)
$$

sufficient conditions are found for the existence of a solution satisfying the conditions

$$
\varphi(u)=c, \quad u(t)>0, \quad u^{\prime}(t)<0 \quad \text { for } t>0,
$$

where $\varphi: C\left([0, a] ; R_{+}\right) \rightarrow R_{+}$is a continuous nondecreasing functional, $c>0$, and $a>0$. MSC: $34 \mathrm{~B} 16 ; 34 \mathrm{~B} 40$

Keywords: differential equation; second order; singular in phase variables; Kneser solution; Kneser problem; nonlinear

\section{Statement of the problem and formulation of the main results}

Suppose

$$
D=\{(t, x, y): t>0, x>0, y<0\}, \quad R_{+}=[0,+\infty[,
$$

and $f: D \rightarrow R_{+}$is a continuous function. Consider the differential equation

$$
u^{\prime \prime}=f\left(t, u, u^{\prime}\right) .
$$

A continuous function $u: R_{+} \rightarrow R_{+}$is said to be the Kneser solution of Eq. (1.1) if it is twice continuously differentiable in the interval $] 0,+\infty[$, and in this interval it satisfies the inequalities

$$
u(t)>0, \quad u^{\prime}(t)<0
$$

and the differential equation (1.1).

๑) 2014 Partsvania and Pưža; licensee Springer. This is an Open Access article distributed under the terms of the Creative Commons Attribution License (http://creativecommons.org/licenses/by/4.0), which permits unrestricted use, distribution, and reproduction in any medium, provided the original work is properly credited. 
In the present paper, we investigate the problem on the existence of a Kneser solution of Eq. (1.1) satisfying the condition

$$
\varphi(u)=c,
$$

where $\varphi: C\left([0, a] ; R_{+}\right) \rightarrow R_{+}$is a continuous, nondecreasing functional, $a>0$, and $c>0$.

It is natural to name this problem the nonlinear Kneser problem since it was first studied by Kneser [1] in the case where Eq. (1.1) and condition (1.2) have the forms

$$
\begin{aligned}
& u^{\prime \prime}=f_{0}(t, u), \\
& u(0)=c,
\end{aligned}
$$

where $f_{0}: R_{+} \times R_{+} \rightarrow R_{+}$is a continuous function. Particularly, in [1] it is proved that if $f_{0}$ is a nondecreasing in the second argument function satisfying the local Lipschitz condition in this argument and $f_{0}(t, 0) \equiv 0$, then for an arbitrarily fixed $c>0$, the differential equation (1.3) has a unique Kneser solution satisfying condition (1.4).

30 years later since Kneser's paper was published, in their study of the problem on the distribution of electrons in a heavy atom, Fermi [2] and Thomas [3] had to investigate the problem analogous to the Kneser one for the concrete second-order differential equation

$$
u^{\prime \prime}=t^{-\frac{1}{2}} u^{\frac{3}{2}}
$$

In particular, they have proved that Eq. (1.5) has a unique solution satisfying the boundary conditions

$$
u(0)=1, \quad \lim _{t \rightarrow+\infty} u(t)=0 .
$$

It is easy to see that a solution of problem (1.5), (1.6) is a Kneser solution of Eq. (1.5) and vice versa, a Kneser solution of that equation, satisfying the initial condition

$$
u(0)=1,
$$

is a solution of problem (1.5), (1.6). Therefore, problem (1.5), (1.6) is equivalent to the Kneser problem for Eq. (1.5) with the initial condition (1.7).

After the papers by Fermi and Thomas were published, many mathematicians have been interested in the Kneser-type problems, and such problems have been investigated in detail for a wide class of differential equations and systems.

Most of the results on the solvability and unique solvability of the Kneser problem for second-order nonlinear differential equations, obtained until the beginning of $50 \mathrm{~s}$ of the last century, are reflected in the monograph by Sansone [4]. From further investigations, first of all the paper by Hartman and Wintner [5], where the Kneser problem for Eq. (1.1) was studied in the case when $f: R_{+} \times R^{2} \rightarrow R_{+}$is a continuous function, should be noted. Kiguradze [6,7] studied the same problem in the case when the function $f:] 0,+\infty\left[\times R^{2} \rightarrow R\right.$ has a nonintegrable singularity in the first argument at the point $t=0$. 
The Kneser problem for singular in a time variable higher-order nonlinear differential equations first was studied by Kiguradze in [8], where the optimal conditions are established for the solvability of the above-mentioned problem (see, [9, Sect. 13] as well). Analogous results were obtained by Kiguradze and Rachůnková [10] for the Kneser problem with a nonlinear initial condition.

Sufficient conditions for the solvability of the Kneser-type problems for nonlinear differential systems were obtained by Chanturia [11], Coffman [12], Hartman and Wintner [13], Kiguradze and Rachůnková [14], and Rachůnková [15, 16].

In all the above-mentioned works, differential equations and systems, not having singularities in phase variables, are considered. The Kneser problem for the differential equation with a singularity in one of the phase variables first was investigated by Kiguradze [17]. However, in this paper we consider not the general differential equation but the EmdenFowler type higher-order differential equation

$$
u^{(n)}=p(t) u^{-\lambda}
$$

As for the general differential equation (1.1) with singularities in phase variables, for it the Kneser problem has been practically unstudied so far. The aim of the present paper is to fill this gap.

In what follows it is assumed that the function $f$ satisfies the inequality

$$
g_{0}(t) \leq x^{\lambda}|y|^{\mu} f(t, x, y) \leq g_{1}(t)
$$

in the domain $D$. Here $\lambda$ and $\mu$ are nonnegative constants, $\lambda+\mu>0$, and $\left.g_{i}:\right] 0,+\infty\left[\rightarrow R_{+}\right.$ $(i=0,1)$ are continuous functions, not equal identically to zero in an arbitrary neighborhood of $+\infty$, i.e., there exists a sequence of positive numbers $\left(t_{k}\right)_{k=1}^{+\infty}$ such that

$$
\lim _{k \rightarrow+\infty} t_{k}=+\infty, \quad g_{i}\left(t_{k}\right)>0 \quad(i=0,1 ; k=1,2, \ldots) .
$$

Consequently, Eq. (1.1) has singularities in phase variables since either

$$
\lim _{x \rightarrow 0} f\left(t_{k}, x, y\right)=+\infty \quad \text { for } y<0(k=1,2, \ldots)
$$

or

$$
\lim _{y \rightarrow 0} f\left(t_{k}, x, y\right)=+\infty \quad \text { for } x>0(k=1,2, \ldots)
$$

Throughout the paper, the following notation and definitions are used.

$$
v=\frac{1+\lambda+\mu}{1+\mu} .
$$

$C([0, a] ; R)$ is the Banach space of continuous functions $u:[0, a] \rightarrow R$ with the norm

$$
\begin{aligned}
& \|u\|_{C}=\max \{|u(t)|: 0 \leq t \leq a\}, \\
& C\left([0, a] ; R_{+}\right)=\{u \in C([0, a] ; R): u(t) \geq 0 \text { for } 0 \leq t \leq a\} .
\end{aligned}
$$


A functional $\varphi: C\left([0, a] ; R_{+}\right) \rightarrow R_{+}$is said to be nondecreasing if for any $u \in C\left([0, a] ; R_{+}\right)$ and $u_{0} \in C\left([0, a] ; R_{+}\right)$the inequality $\varphi\left(u+u_{0}\right) \geq \varphi(u)$ holds.

For any $x \in R_{+}$, we put $\varphi(x)=\varphi(u)$, where $u(t) \equiv x$.

A Kneser solution $u$ of Eq. (1.1) is called vanishing at infinity if $\lim _{t \rightarrow+\infty} u(t)=0$, and it is called remote from zero if $\lim _{t \rightarrow+\infty} u(t)>0$.

Theorem 1.1 If Eq. (1.1) has a Kneser solution $u$, then

$$
\int_{t}^{+\infty} g_{0}(s) d s<+\infty \text { for } t>0, \quad \int_{0}^{+\infty}\left(\int_{t}^{+\infty} g_{0}(s) d s\right)^{\frac{1}{1+\mu}} d t<+\infty
$$

and

$$
u(t)>v_{0}(t ; \delta) \quad \text { for } t \geq 0,
$$

where

$$
\begin{aligned}
& v_{0}(t ; \delta)=\left[\delta^{v}+(1+\mu)^{\frac{1}{1+\mu}} v \int_{t}^{+\infty}\left(\int_{s}^{+\infty} g_{0}(x) d x\right)^{\frac{1}{1+\mu}} d s\right]^{\frac{1}{v}}, \\
& \delta=\lim _{t \rightarrow+\infty} u(t) .
\end{aligned}
$$

Corollary 1.1 If condition (1.11) holds and

$$
c<\varphi\left(v_{0}(\cdot ; 0)\right)
$$

then problem (1.1), (1.2) has no Kneser solution.

\section{Theorem 1.2 If}

$$
\int_{t}^{+\infty} g_{1}(s) d s<+\infty \quad \text { for } t>0, \quad \int_{0}^{+\infty}\left(\int_{t}^{+\infty} g_{1}(s) d s\right)^{\frac{1}{1+\mu}} d t<+\infty,
$$

then for any positive number $\delta$ Eq. (1.1) has at least one Kneser solution satisfying equality (1.14).

Theorem 1.3 If along with (1.11) the condition

$$
\int_{t}^{+\infty} \frac{g_{1}(s)}{v_{0}^{\lambda}(s ; 0)} d s<+\infty \quad \text { for } t>0, \quad \int_{0}^{+\infty}\left(\int_{t}^{+\infty} \frac{g_{1}(s)}{v_{0}^{\lambda}(s ; 0)} d s\right)^{\frac{1}{1+\mu}} d t<+\infty
$$

is satisfied, then Eq. (1.1) has at least one vanishing at infinity Kneser solution.

According to Corollary 1.1, for small $c$ problem (1.1), (1.2) has no Kneser solution. Thus we can expect the solvability of that problem only for large $c$.

Suppose that condition (1.16) holds. Then obviously condition (1.11) is satisfied as well. We introduce the function

$$
v_{1}(t ; \delta)=\delta+\int_{t}^{+\infty}\left[(1+\mu) \int_{s}^{+\infty} \frac{g_{1}(x)}{v_{0}^{\lambda}(x ; \delta)} d x\right]^{\frac{1}{1+\mu}} d s \quad \text { for } t \geq 0, \delta>0,
$$


and the number

$$
c_{0}=\inf \left\{\varphi\left(v_{1}(\cdot ; \delta)\right): \delta>0\right\} .
$$

Theorem 1.4 Let the function $g_{1}$ satisfy condition (1.16), and

$$
\lim _{x \rightarrow+\infty} \varphi(x)=+\infty
$$

If, moreover,

$$
c>c_{0}
$$

then problem (1.1), (1.2) has at least one Kneser solution.

Remark 1.1 In the case, where conditions (1.16) hold and

$$
c \in\left[\varphi\left(v_{0}(\cdot ; 0)\right), c_{0}\right]
$$

the question on the existence of a Kneser solution of problem (1.1), (1.2) remains open.

Consider now the case where

$$
g_{1}(t) \equiv \ell g_{0}(t), \quad \ell=\text { const } \geq 1,
$$

i.e., the case where inequality (1.8) has the form

$$
g_{0}(t) \leq x^{\lambda}|y|^{\mu} f(t, x, y) \leq \ell g_{0}(t)
$$

From Theorems 1.1, 1.2, and 1.4 we immediately have the following corollary.

Corollary 1.2 Let the function $g_{1}$ satisfy identity (1.22), and let the functional $\varphi$ satisfy condition (1.20). Then the following assertions are equivalent:

(i) the function $g_{0}$ satisfies conditions (1.11);

(ii) Eq. (1.1) has at least one remote from zero Kneser solution;

(iii) for any $\delta>0$, problem (1.1), (1.14) has at least one Kneser solution;

(iv) for any sufficiently large $c>0$, problem (1.1), (1.2) has at least one Kneser solution.

The following statement is also valid.

Corollary 1.3 Let the function $g_{1}$ satisfy identity (1.22), and the functional $\varphi$ satisfy condition (1.20). Let, moreover, there exist numbers $\alpha$ and $\beta$ such that

$$
\begin{aligned}
& \liminf _{t \rightarrow 0}\left(t^{\alpha} g_{0}(t)\right)>0, \quad \limsup _{t \rightarrow 0}\left(t^{\alpha} g_{0}(t)\right)<+\infty, \\
& \liminf _{t \rightarrow+\infty}\left(t^{\beta} g_{0}(t)\right)>0, \quad
\end{aligned}
$$

Then the following assertions are equivalent:

(i) $\alpha<2+\mu, \beta>2+\mu$;

(ii) Eq. (1.1) has at least one remote from zero Kneser solution; 
(iii) Eq. (1.1) has at least one vanishing at infinity Kneser solution;

(iv) for any $\delta>0$, problem (1.1), (1.14) has at least one Kneser solution;

(v) for any sufficiently large $c>0$, problem (1.1), (1.2) has at least one Kneser solution.

Remark 1.2 In Theorem 1.4 and its corollaries it can be assumed, for example, that

$$
\varphi(u)=\int_{0}^{a} \psi(u(s)) d \sigma(s),
$$

where $\psi: R_{+} \rightarrow R_{+}$is a continuous, nondecreasing function, and $\sigma:[0, a] \rightarrow R$ is a nondecreasing function such that

$$
\lim _{x \rightarrow+\infty} \psi(x)=+\infty, \quad \sigma(a)-\sigma(0)>0
$$

Remark 1.3 The above-formulated theorems and their corollaries cover the case, where the function $f$ has a nonintegrable singularity in a time variable at the point $t=0$. Indeed, if conditions (1.23) hold, where $1<\alpha<2+\mu$, then

$$
\int_{0}^{t} f(s, x, y) d s=+\infty \quad \text { for }(t, x, y) \in D
$$

\section{Auxiliary propositions}

\subsection{Lemmas on a priori estimates}

Consider the differential inequalities

$$
\left|u^{\prime}(t)\right|^{\mu} u^{\prime \prime}(t) \geq g_{0}(t) u^{-\lambda}(\tau(t))
$$

and

$$
g_{0}(t) u^{-\lambda}(\tau(t)) \leq\left|u^{\prime}(t)\right|^{\mu} u^{\prime \prime}(t) \leq g_{1}(t) u^{-\lambda}(\tau(t))
$$

Everywhere in this section it is assumed that $\lambda$ and $\mu$ are nonnegative constants, $\tau: R_{+} \rightarrow$ $R_{+}$is a continuous function such that

$$
\tau(t) \geq t \quad \text { for } t \in R_{+},
$$

and $\left.g_{i}:\right] 0,+\infty\left[\rightarrow R_{+}(i=0,1)\right.$ are continuous functions, not equal identically to zero in an arbitrary neighborhood of $+\infty$, i.e., there exists a sequence of positive numbers $\left(t_{k}\right)_{k=1}^{+\infty}$ such that condition (1.9) is satisfied.

A continuous function $\left.u: R_{+} \rightarrow\right] 0,+\infty$ [ is said to be the Kneser solution of the differential inequality (2.1) (of the differential inequality (2.2)) if it is twice continuously differentiable in the interval $] 0,+\infty$ [ and in this interval along with the inequality $u^{\prime}(t)<0$ satisfies the differential inequality (2.1) (the differential inequality (2.2)).

Lemma 2.1 If the differential inequality (2.1) has a Kneser solution $u$, then the function $g_{0}$ satisfies condition (1.11), and $u$ admits estimate (1.12), where $v_{0}$ is the function given by equality (1.13), and $v, \delta$ are numbers given by equalities (1.10) and (1.14). 
Proof In view of (2.3) and the fact that $u$ is a Kneser solution, from (2.1) we find

$$
\begin{aligned}
\left|u^{\prime}(t)\right|^{1+\mu} & =(1+\mu) \int_{t}^{+\infty} u^{\lambda}(\tau(x))\left|u^{\prime}(x)\right|^{\mu} u^{\prime \prime}(x) u^{-\lambda}(\tau(x)) d x \\
& >(1+\mu) u^{-\lambda}(t) \int_{t}^{+\infty} u^{\lambda}(\tau(x))\left|u^{\prime}(x)\right|^{\mu} u^{\prime \prime}(x) d x \geq(1+\mu) u^{-\lambda}(t) \int_{t}^{+\infty} g_{0}(x) d x
\end{aligned}
$$

and, consequently,

$$
-u^{\prime}(t)[u(t)]^{\frac{\lambda}{1+\mu}}>\left[(1+\mu) \int_{t}^{+\infty} g_{0}(x) d x\right]^{\frac{1}{1+\mu}} \text { for } t>0
$$

If we integrate this inequality from $t$ to $+\infty$, then due to equalities (1.10) and (1.14), we obtain

$$
[u(t)]^{\nu}>\delta^{\nu}+(1+\mu)^{\frac{1}{1+\mu}} v \int_{t}^{+\infty}\left(\int_{s}^{+\infty} g_{0}(x) d x\right)^{\frac{1}{1+\mu}} d s \quad \text { for } t \geq 0 .
$$

Therefore condition (1.11) is satisfied and the function $u$ admits estimate (1.12).

Let $b \in] 0,+\infty$ [, and along with (2.3) let the inequality

$$
\tau(t) \leq b \quad \text { for } 0 \leq t \leq b
$$

be fulfilled. A continuous function $u:[0, b] \rightarrow] 0,+\infty[$ is said to be $a$ Kneser solution of the differential inequality (2.1) (of the differential inequality (2.2)) in the interval $[0, b]$ if it is continuously differentiable in the interval $] 0, b]$ and in this interval along with the inequality $u^{\prime}(t)<0$ satisfies the differential inequality (2.1) (the differential inequality (2.2)).

The following lemma can be proved analogously to Lemma 2.1.

Lemma 2.2 Let inequality (2.4) be fulfilled and the differential inequality (2.1) in the interval $[0, b]$ have a Kneser solution $u$. Then

$$
\int_{0}^{b}\left(\int_{s}^{b} g_{0}(x) d x\right)^{\frac{1}{1+\mu}} d s<+\infty
$$

and that solution admits the estimate

$$
u(t)>w_{0}(t ; \delta, b) \quad \text { for } 0 \leq t \leq b,
$$

where $\delta=u(b)$ and

$$
w_{0}(t ; \delta, b)=\left[\delta^{\nu}+(1+\mu)^{\frac{1}{1+\mu}} v \int_{t}^{b}\left(\int_{s}^{b} g_{0}(x) d x\right)^{\frac{1}{1+\mu}} d s\right]^{\frac{1}{\nu}} \quad \text { for } 0 \leq t \leq b .
$$

Lemma 2.3 Let along with (2.4) the condition

$$
\int_{0}^{b}\left(\int_{s}^{b} g_{1}(x) d x\right)^{\frac{1}{1+\mu}} d s<+\infty
$$


be fulfilled, and let the differential inequality $(2.2)$ in the interval $[0, b]$ have a Kneser solution $u$. Then this solution along with (2.6) admits the estimates

$$
\begin{aligned}
u(t) \leq w(t ; \delta, \varepsilon, b) \quad \text { for } 0 \leq t \leq b, & \\
{\left[\varepsilon^{1+\mu}+w^{-\lambda}(0 ; \delta, \varepsilon, b) \int_{t}^{b} g_{0}(s) d s\right]^{\frac{1}{1+\mu}} } & \leq-u^{\prime}(t) \\
& \leq-w^{\prime}(t ; \delta, \varepsilon, b) \text { for } 0<t<b,
\end{aligned}
$$

where $\delta=u(b), \varepsilon=\left|u^{\prime}(b)\right|$, and

$$
w(t ; \delta, \varepsilon, b)=\delta+\int_{t}^{b}\left[\varepsilon^{1+\mu}+(1+\mu) \int_{s}^{b} \frac{g_{1}(x) d x}{w_{0}^{\lambda}(\tau(x) ; \delta, b)}\right]^{\frac{1}{1+\mu}} d s
$$

for $0 \leq t \leq b$.

Proof First note that the validity of condition (2.8) guarantees the validity of condition (2.5). On the other hand, by Lemma 2.2 the function $u$ admits estimate (2.6). By virtue of this estimate and the fact that $u$ is a Kneser solution, (2.2) and (2.11) yield

$$
\begin{aligned}
\left|u^{\prime}(t)\right|^{1+\mu} & =\varepsilon^{1+\mu}+(1+\mu) \int_{t}^{b}\left|u^{\prime}(x)\right|^{\mu} u^{\prime \prime}(x) d x \\
& \geq \varepsilon^{1+\mu}+\int_{t}^{b} g_{0}(x) u^{-\lambda}(\tau(x)) d x \\
& \geq \varepsilon^{1+\mu}+u^{-\lambda}(0) \int_{t}^{b} g_{0}(x) d x \quad \text { for } 0<t \leq b, \\
\left|u^{\prime}(t)\right|^{1+\mu} & \leq \varepsilon^{1+\mu}+(1+\mu) \int_{t}^{b} g_{1}(x) u^{-\lambda}(x) d x \\
& \leq \varepsilon^{1+\mu}+(1+\mu) \int_{t}^{b} \frac{g_{1}(x)}{w_{0}^{\lambda}(x ; \delta, b)} d x=\left[-w^{\prime}(t ; \delta, \varepsilon, b)\right]^{1+\mu},
\end{aligned}
$$

and, consequently,

$$
-u^{\prime}(t) \leq-w^{\prime}(t ; \delta, \varepsilon, b) \quad \text { for } 0<t \leq b .
$$

Integration of this inequality from $t$ to $b$ results in estimate (2.9).

If along with (2.9) we take into account inequalities (2.12) and (2.13), then the validity of estimate (2.10) becomes evident.

\subsection{Lemma on the solvability of a nonlinear Kneser problem on a finite interval}

Let $b>a$. Consider the problem on the existence of a Kneser solution of Eq. (1.1) in the interval $[0, b]$ satisfying condition (1.2).

If condition (2.8) holds, then for any $\delta>0, \varepsilon>0$, and $t \in[0, b]$, we assume

$$
w_{1}(t ; \delta, \varepsilon, b)=\delta+\int_{t}^{b}\left[\varepsilon^{1+\mu}+(1+\mu) \int_{s}^{b} \frac{g_{1}(x) d x}{w_{0}^{\lambda}(x ; \delta, b)}\right]^{\frac{1}{1+\mu}} d s .
$$


Lemma 2.4 Let condition (2.8) be fulfilled and there exist numbers $\varepsilon>0, \delta_{0}>0$, and $\delta^{*}>\delta_{0}$ such that

$$
\varphi\left(\delta^{*}\right)>c
$$

and

$$
\varphi\left(w_{1}\left(\cdot ; \delta_{0}, \varepsilon, b\right)\right)<c .
$$

Then problem (1.1), (1.2) has a Kneser solution $u$ in the interval $[0, b]$ such that

$$
\delta_{0} \leq u(b) \leq \delta^{*}, \quad u^{\prime}(b)=-\varepsilon .
$$

Proof For arbitrarily fixed $\delta \in\left[\delta_{0}, \delta^{*}\right]$ and natural number $k$, we consider the Cauchy problem

$$
\begin{aligned}
& u^{\prime \prime}(t)=\left|u^{\prime}(t)\right|^{-\mu} f_{0}\left(t, u\left(\tau_{k}(t)\right), u^{\prime}\left(\tau_{k}(t)\right)\right), \\
& u(b)=\delta, \quad u^{\prime}(b)=-\varepsilon,
\end{aligned}
$$

where

$$
\begin{aligned}
& f_{0}(t, x, y)=|y|^{\mu} f(t, x, y), \\
& \tau_{k}(t)= \begin{cases}t+\frac{b}{2 k} & \text { for } 0 \leq t \leq b-\frac{b}{2 k}, \\
b & \text { for } b-\frac{b}{2 k}<t \leq b .\end{cases}
\end{aligned}
$$

By virtue of conditions (1.8), (2.8) and equalities (2.20), (2.21), problem (2.18), (2.19) has a unique solution in the interval $[0, b]$, which is a Kneser solution of the differential inequality (2.2) as well, where $\tau(x) \equiv \tau_{k}(x)$. Denote this solution by $u_{k}(t ; \delta)$. It is clear that the function $(t, \delta) \rightarrow u_{k}(t ; \delta)$ is continuous on $[0, b] \times\left[\delta_{0}, \delta^{*}\right]$, and on $] 0, b\left[\times\left[\delta_{0}, \delta^{*}\right]\right.$ it satisfies the inequalities

$$
u_{k}(t ; \delta)>\delta, \quad u_{k}^{\prime}(t ; \delta) \leq-\varepsilon
$$

On the other hand, by Lemma 2.3 , on $] 0, b] \times\left[\delta_{0}, \delta^{*}\right]$ this function admits the estimates

$$
\begin{aligned}
& u_{k}(t ; \delta) \leq w_{1 k}(t ; \delta, \varepsilon, b) \leq r, \\
& -u_{k}^{\prime}(t ; \delta) \leq-w_{1 k}^{\prime}(t ; \delta, \varepsilon, b) \leq-w_{10}^{\prime}(t),
\end{aligned}
$$

where

$$
\begin{aligned}
& w_{1 k}(t ; \delta, \varepsilon, b)=\delta+\int_{t}^{b}\left[\varepsilon^{1+\mu}+(1+\mu) \int_{s}^{b} \frac{g_{1}(x) d x}{w_{0}^{\lambda}\left(\tau_{k}(x) ; \delta, b\right)}\right]^{\frac{1}{1+\mu}} d s, \\
& w_{10}(t)=\delta^{*}+\int_{t}^{b}\left[\varepsilon^{1+\mu}+(1+\mu) \delta_{0}^{-\lambda} \int_{s}^{b} g_{1}(x) d x\right]^{\frac{1}{1+\mu}} d s, \\
& r=w_{10}(0) .
\end{aligned}
$$


Due to (2.8) and (2.21), the equality

$$
\lim _{k \rightarrow+\infty} w_{1 k}(t ; \delta, \varepsilon, b)=w_{1}(t ; \delta, \varepsilon, b)
$$

is satisfied uniformly with respect to $(t, \delta) \in[0, b] \times\left[\delta_{0}, \delta^{*}\right]$. Thus from (2.16) it follows the inequality

$$
\varphi\left(w_{1 k}\left(\cdot ; \delta_{0}, \varepsilon, b\right)\right)<c \text { for } k \geq k_{0} \text {, }
$$

where $k_{0}$ is a sufficiently large natural number.

Let $k \geq k_{0}$. Suppose

$$
\psi_{k}(\delta)=\varphi\left(u_{k}(\cdot ; \delta)\right) \quad \text { for } \delta_{0} \leq \delta \leq \delta^{*}
$$

From the continuity of $\varphi$ and $u_{k}$ it follows that $\psi_{k}$ is a continuous on $\left[\delta_{0}, \delta^{*}\right]$ function. On the other hand, by virtue of estimates (2.22) and (2.23), inequalities (2.15) and (2.25) yield the inequalities

$$
\psi_{k}\left(\delta_{0}\right)<c, \quad \psi_{k}\left(\delta^{*}\right)>c,
$$

since $\varphi$ is a nondecreasing functional. From these inequalities it follows the existence of a number $\left.\delta_{k} \in\right] \delta_{0}, \delta^{*}\left[\right.$ such that $\psi_{k}\left(\delta_{k}\right)=c$. Consequently,

$$
\begin{aligned}
& u_{k}^{\prime \prime}\left(t ; \delta_{k}\right)=\left|u_{k}^{\prime}\left(t ; \delta_{k}\right)\right|^{-\mu} f_{0}\left(t, u_{k}\left(\tau_{k}(t) ; \delta_{k}\right), u_{k}^{\prime}\left(\tau_{k}(t) ; \delta_{k}\right)\right) \quad \text { for } 0<t \leq b, \\
& \varphi\left(u_{k}\left(\cdot ; \delta_{k}\right)\right)=c
\end{aligned}
$$

In view of estimates (2.22)-(2.24), for every natural $k \geq k_{0}$, the inequalities

$$
\delta_{0}<\delta_{k} \leq u_{k}\left(t ; \delta_{k}\right) \leq r, \quad 0 \leq u_{k}\left(0 ; \delta_{k}\right)-u_{k}\left(t ; \delta_{k}\right) \leq w_{10}(0)-w_{10}(t)
$$

hold in the interval $[0, b]$, and the inequalities

$$
\begin{aligned}
& \varepsilon \leq-u_{k}^{\prime}\left(t ; \delta_{k}\right) \leq-w_{10}^{\prime}(t), \\
& 0 \leq u_{k}^{\prime \prime}\left(t ; \delta_{k}\right) \leq \delta_{0}^{-\lambda} \varepsilon^{-\lambda} g_{1}(t)
\end{aligned}
$$

hold in the interval $] 0, b]$. From these inequalities it follows that the sequence $\left(u_{k}\left(\cdot ; \delta_{k}\right)\right)_{k=k_{0}}^{+\infty}$ is uniformly bounded and equicontinuous on $[0, b]$, and the sequence $\left(u_{k}^{\prime}\left(\cdot ; \delta_{k}\right)\right)_{k=k_{0}}^{+\infty}$ is uniformly bounded and equicontinuous on every closed interval contained in $] 0, b]$.

According to the Arzelà-Ascoli lemma, without loss of generality it can be assumed that $\left(u_{k}\left(\cdot ; \delta_{k}\right)\right)_{k=k_{0}}^{+\infty}$ is uniformly converging on the interval $[0, b]$, and $\left(u_{k}^{\prime}\left(\cdot ; \delta_{k}\right)\right)_{k=k_{0}}^{+\infty}$ is uniformly converging on every closed interval contained in $] 0, b]$. Then, by virtue of notation (2.20), (2.21) and estimates (2.28), (2.29), from equality (2.26) it follows that the sequence $\left(u_{k}^{\prime \prime}\left(\cdot ; \delta_{k}\right)\right)_{k=k_{0}}^{+\infty}$ is also uniformly converging on every closed interval contained in $\left.] 0, b\right]$.

Suppose

$$
u(t)=\lim _{k \rightarrow+\infty} u_{k}\left(t ; \delta_{k}\right) \quad \text { for } 0 \leq t \leq b
$$


It is evident that the function $u$ is continuous on $[0, b]$ and twice continuously differentiable on $] 0, b]$. Moreover,

$$
\begin{aligned}
& u(t)=\lim _{k \rightarrow+\infty} u_{k}\left(\tau_{k}(t) ; \delta_{k}\right) \quad \text { for } 0 \leq t \leq b, \\
& u^{\prime}(t)=\lim _{k \rightarrow+\infty} u_{k}^{\prime}\left(t ; \delta_{k}\right)=\lim _{k \rightarrow+\infty} u_{k}^{\prime}\left(\tau_{k}(t) ; \delta_{k}\right), \\
& u^{\prime \prime}(t)=\lim _{k \rightarrow+\infty} u_{k}^{\prime \prime}\left(t ; \delta_{k}\right) \quad \text { for } 0<t \leq b,
\end{aligned}
$$

and conditions (2.17) are satisfied.

If now we pass to the limit in equalities (2.26) and (2.27) as $k \rightarrow+\infty$ and take into account estimates (2.28) and (2.29), then it becomes evident that $u$ is a Kneser solution of problem (1.1), (1.2) in the interval $[0, b]$.

\subsection{Lemma on the solvability of the Cauchy problem on a finite interval}

Lemma 2.5 Let $b>0$ and condition (2.8) be fulfilled. Then, for arbitrarily fixed $\delta>0$ and $\varepsilon>0$, problem (1.1), (2.19) has at least one Kneser solution in the interval $[0, b]$ and every such solution admits the estimates

$$
\begin{aligned}
& w_{0}(t ; \delta, b) \leq u(t) \leq w_{1}(t ; \delta, \varepsilon, b) \quad \text { for } 0 \leq t \leq b, \\
& {\left[\varepsilon^{1+\mu}+w_{1}^{-\lambda}(0 ; \delta, \varepsilon, b) \int_{t}^{b} g_{0}(s) d s\right]^{\frac{1}{1+\mu}} \leq-u^{\prime}(t)} \\
& \leq-w_{1}^{\prime}(t ; \delta, \varepsilon, b) \text { for } 0<t<b,
\end{aligned}
$$

where $w_{0}$ and $w_{1}$ are functions given by equalities (2.7) and (2.14).

Proof According to the Peano theorem, the continuity of the function $f: D \rightarrow R_{+}$guarantees the local solvability of problem (1.1), (2.19). Let $u$ be any maximally extended solution of that problem defined on some interval $\left.] b_{0}, b\right]$. Then on this interval the inequalities

$$
u(t) \geq \delta, \quad u^{\prime}(t) \leq-\varepsilon, \quad u^{\prime \prime}(t) \leq 0
$$

hold. Moreover, either $b_{0}=0$, or $b_{0}>0$ and

$$
\lim _{t \rightarrow b_{0}} u^{\prime}(t)=-\infty
$$

By virtue of conditions (1.8) and (2.32), we have

$$
0 \leq u^{\prime \prime}(t)\left|u^{\prime}(t)\right|^{\mu} \leq \delta^{-\lambda} g_{1}(t) \quad \text { for } b_{0}<t \leq b .
$$

Hence, in view of (2.19), we find

$$
\left|u^{\prime}(t)\right| \leq g(t) \quad \text { for } b_{0}<t \leq b,
$$

where

$$
g(t)=\left[\varepsilon^{1+\mu}+(1+\mu) \delta^{-\lambda} \int_{t}^{b} g_{1}(s) d s\right]^{\frac{1}{1+\mu}} \text { for } 0<t \leq b,
$$


and, as it follows from (2.8),

$$
\int_{0}^{b} g(s) d s<+\infty
$$

If we assume that $b_{0}>0$, then from estimate (2.34) we find

$$
\lim _{t \rightarrow b_{0}}\left|u^{\prime}(t)\right| \leq g\left(b_{0}\right)<+\infty
$$

But this contradicts equality (2.33). The contradiction obtained proves that $b_{0}=0$.

Due to (2.34) and (2.35), the function $u$ has a finite right-hand limit at the point 0 . If we put

$$
u(0)=\lim _{t \rightarrow 0} u(t)
$$

then it becomes obvious that $u$ is a Kneser solution of problem (1.1), (2.19) in the interval $[0, b]$.

According to condition (1.8), the function $u$ is a Kneser solution of the differential inequality (2.2) as well, where $\tau(t) \equiv t$. By Lemma 2.3, this solution admits estimates (2.6), (2.9), (2.10). On the other hand, when $\tau(t) \equiv t$, from (2.11) and (2.14) it follows that

$$
w(t ; \delta, \varepsilon, b) \equiv w_{1}(t ; \delta, \varepsilon, b) .
$$

Therefore estimates (2.30) and (2.31) are valid.

\subsection{Lemma on the existence of a remote from zero Kneser solution of Eq. (1.1)}

Lemma 2.6 Let $\delta$ be a positive number, and let $\left(b_{k}\right)_{k=1}^{+\infty},\left(\delta_{k}\right)_{k=1}^{+\infty}$, and $\left(\varepsilon_{k}\right)_{k=1}^{+\infty}$ be sequences of positive numbers such that

$$
\begin{aligned}
& \lim _{k \rightarrow+\infty} b_{k}=+\infty, \quad \lim _{k \rightarrow+\infty} \delta_{k}=\delta, \\
& \varepsilon_{k} b_{k}<\frac{1}{k} \quad(k=1,2, \ldots),
\end{aligned}
$$

and for any natural $k$, the differential equation (1.1) has a Kneser solution $u_{k}$ in the interval $\left[0, b_{k}\right]$ satisfying the conditions

$$
u_{k}\left(b_{k}\right)=\delta_{k}, \quad u_{k}^{\prime}\left(b_{k}\right)=-\varepsilon_{k} \text {. }
$$

Let, moreover, condition (1.16) be satisfied. Then the sequence $\left(u_{k}\right)_{k=1}^{+\infty}$ contains a uniformly converging on every finite interval of $R_{+}$subsequence, whose limit is a Kneser solution of problem (1.1), (1.14).

Proof According to Lemma 2.5 and condition (1.16), for any natural $k$ the inequality

$$
w_{0}\left(t ; \delta_{k}, b_{k}\right) \leq u_{k}(t) \leq w_{1}\left(t ; \delta_{k}, \varepsilon_{k}, b_{k}\right)
$$


holds in the interval $\left[0, b_{k}\right]$, and the inequality

$$
\left[\varepsilon_{k}^{1+\mu}+w_{1}^{-\lambda}\left(0 ; \delta_{k}, \varepsilon_{k}, b_{k}\right) \int_{t}^{b_{k}} g_{0}(s) d s\right]^{\frac{1}{1+\mu}} \leq-u_{k}^{\prime}(t) \leq-w_{1}^{\prime}\left(t ; \delta_{k}, \varepsilon_{k}, b_{k}\right)
$$

holds in the interval $\left.] 0, b_{k}\right]$. Hence, by virtue of conditions (1.8), (1.16), and (2.37), we have

$$
\begin{aligned}
& \delta_{k} \leq u_{k}(t) \leq \delta_{k}+\frac{1}{k}+\int_{t}^{+\infty} h(s) d s \leq r \quad \text { for } 0 \leq t \leq b_{k}, \\
& h_{k}(t) \leq-u_{k}^{\prime}(t) \leq \frac{1}{k}+h(t) \quad \text { for } 0<t \leq b_{k}, \\
& 0 \leq u_{k}^{\prime \prime}(t) \leq \delta_{0}^{-\lambda} h_{k}^{-\mu}(t) g_{1}(t) \quad \text { for } 0<t \leq b_{k},
\end{aligned}
$$

where

$$
\begin{aligned}
& \delta_{0}=\inf \left\{\delta_{k}: k=1,2, \ldots\right\}>0, \quad r=1+r_{0}+\sup \left\{\delta_{k}: k=1,2, \ldots\right\}, \\
& r_{0}=\int_{0}^{+\infty} h(s) d s<+\infty \\
& h(t)=\left[(1+\mu) \delta_{0}^{-1} \int_{t}^{+\infty} g_{1}(s) d s\right]^{\frac{1}{1+\mu}}, \\
& h_{k}(t)=\left[\varepsilon_{k}^{1+\mu}+r^{-\lambda} \int_{t}^{b_{k}} g_{0}(s) d s\right]^{\frac{1}{1+\mu}} .
\end{aligned}
$$

On the other hand, in view of (1.9) and (2.36), it is clear that

$$
\lim _{k \rightarrow+\infty} h_{k}(t)=h_{0}(t)>0 \quad \text { for } 0<t<+\infty
$$

where

$$
h_{0}(t)=\left[r^{-\lambda} \int_{t}^{+\infty} g_{0}(s) d s\right]^{\frac{1}{1+\mu}}
$$

Let $b>0$ and $\left.b_{0} \in\right] 0, b\left[\right.$ be arbitrary numbers, and let $k_{0}$ and $b^{*}>b$ be such large numbers that

$$
\int_{b}^{b^{*}} g_{0}(s) d s>0, \quad b_{k}>b^{*} \text { for } k \geq k_{0} .
$$

Then in view of inequalities (2.39)-(2.41), the sequence $\left(u_{k}\right)_{k=k_{0}}^{+\infty}$ is uniformly bounded on $[0, b]$, and the sequences $\left(u_{k}^{\prime}\right)_{k=k_{0}}^{+\infty}$ and $\left(u_{k}^{\prime \prime}\right)_{k=k_{0}}^{+\infty}$ are uniformly bounded on $\left[b_{0}, b\right]$. Hence by the Arzelà-Ascoli lemma and conditions (2.40), (2.42) it follows the existence of a subsequence $\left(u_{k_{m}}\right)_{m=1}^{+\infty}$ of the sequence $\left(u_{k}\right)_{k=k_{0}}^{+\infty}$ such that $\left(u_{k_{m}}\right)_{m=1}^{+\infty}$ is uniformly converging on every finite interval contained in $R_{+}$, and $\left(u_{k_{m}}^{\prime}\right)_{m=1}^{+\infty}$ is uniformly converging on every finite closed interval contained in $] 0,+\infty[$. On the other hand, by virtue of conditions (2.39), (2.40), and (2.43), from the equalities

$$
u_{k_{m}}^{\prime \prime}(t)=f\left(t, u_{k_{m}}(t), u_{k_{m}}^{\prime}(t)\right) \quad \text { for } 0<t \leq b_{k_{m}}(m=1,2, \ldots)
$$


it follows that the sequence $\left(u_{k_{m}}^{\prime \prime}\right)_{m=1}^{+\infty}$ is also uniformly converging on every finite closed interval contained in $] 0,+\infty[$.

Suppose

$$
u(t)=\lim _{m \rightarrow+\infty} u_{k_{m}}(t) \quad \text { for } t \in R_{+} .
$$

According to the above said, the function $u$ is continuous on $R_{+}$, twice continuously differentiable on $] 0,+\infty[$, and

$$
u^{\prime}(t)=\lim _{m \rightarrow+\infty} u_{k_{m}}^{\prime}(t), \quad u^{\prime \prime}(t)=\lim _{m \rightarrow+\infty} u_{k_{m}}^{\prime \prime}(t) \quad \text { for } 0<t<+\infty .
$$

Thus from (2.36), (2.39), (2.40), and (2.45) it follows that $u$ is a Kneser solution of problem (1.1), (1.14).

\section{Proof of the main results}

Proof of Theorem 1.1 Let $u$ be a Kneser solution of Eq. (1.1). Then, by condition (1.8), this function is a Kneser solution of the differential inequality $(2.1)$ as well, where $\tau(t) \equiv t$. Due to Lemma 2.1, the function $g_{0}$ satisfies condition (1.11), and $u$ admits estimate (1.12).

Proof of Corollary 1.1 Assume the contrary that problem (1.1), (1.2) has a Kneser solution $u$. Then, by Theorem 1.1, the function $u$ admits estimate (1.12), where $\delta$ is a number, given by equality (1.14). Thus (1.2) yields

$$
c \geq \varphi\left(v_{0}(\cdot ; \delta)\right) \geq \varphi\left(v_{0}(\cdot ; 0)\right)
$$

But this inequality contradicts inequality (1.15). The contradiction obtained proves the validity of the corollary.

Proof of Theorem 1.2 Let $\delta_{k}=\delta(k=1,2, \ldots)$, and let $\left(b_{k}\right)_{k=1}^{+\infty}$ and $\left(\varepsilon_{k}\right)_{k=1}^{+\infty}$ be sequences of positive numbers satisfying conditions (2.36) and (2.37). By Lemma 2.5 and condition (1.16), for any natural $k$ the differential equation (1.1) has a Kneser solution in the interval $\left[0, b_{k}\right]$ satisfying conditions (2.38). Hence, by virtue of Lemma 2.6, it follows the existence of a Kneser solution of problem (1.1), (1.14).

Proof of Theorem 1.3 Conditions (1.11) and (1.17) imply condition (1.16), which by Theorem 1.2 guarantees the existence of a Kneser solution of problem (1.1), (1.14) for any $\delta>0$. Consequently, for any natural $k$, the differential equation (1.1) has a Kneser solution $u_{k}$ such that

$$
\lim _{t \rightarrow+\infty} u_{k}(t)=\frac{1}{k}
$$

On the other hand, by virtue of Theorem 1.1, each $u_{k}$ satisfies the estimate

$$
u_{k}(t)>v_{0}\left(t ; \frac{1}{k}\right)>v_{0}(t ; 0) \quad \text { for } t \geq 0 \text {. }
$$


This estimate, due to conditions (1.8), (1.9), (1.17), yields

$$
\begin{aligned}
& 0<v_{0}(t ; 0)<u_{k}(t)<\frac{1}{k}+v_{1}(t ; 0) \leq r \quad \text { for } t \geq 0, \\
& 0<h_{0}(t)<-u_{k}^{\prime}(t)<v_{1}^{\prime}(t ; 0) \quad \text { for } t>0, \\
& 0 \leq u_{k}^{\prime \prime}(t) \leq h_{0}^{-\lambda}(t) v_{0}^{-\lambda}(t ; 0) g_{1}(t) \quad \text { for } t>0,
\end{aligned}
$$

where $r=1+v_{1}(0 ; 0)$, while $v_{1}$ and $h_{0}$ are functions given by equalities (1.18) and (2.44).

By virtue of the Arzelà-Ascoli lemma and the equality

$$
u_{k}^{\prime \prime}(t)=f_{k}\left(t, u_{k}(t), u_{k}^{\prime}(t)\right) \quad \text { for } t>0
$$

from estimates (3.1)-(3.3) it follows the existence of a subsequence $\left(u_{k_{m}}\right)_{m=1}^{+\infty}$ of the sequence $\left(u_{k}\right)_{k=1}^{+\infty}$ such that $\left(u_{k_{m}}\right)_{m=1}^{+\infty}$ is uniformly converging on every finite closed interval contained in $R_{+}$, and $\left(u_{k_{m}}^{\prime}\right)_{m=1}^{+\infty}$ and $\left(u_{k_{m}}^{\prime \prime}\right)_{m=1}^{+\infty}$ are uniformly converging on every finite closed interval contained in $] 0,+\infty[$.

If we assume

$$
u(t)=\lim _{m \rightarrow+\infty} u_{k_{m}}(t) \text { for } t \geq 0
$$

then in view of conditions (3.1), (3.2), (3.4) we find

$$
\begin{aligned}
& 0<v_{0}(t ; 0) \leq u(t) \leq v_{1}(t ; 0), \\
& u^{\prime}(t)>0, \quad u^{\prime \prime}(t)=f\left(t, u(t), u^{\prime}(t)\right) \text { for } t>0 .
\end{aligned}
$$

Therefore $u$ is a vanishing at infinity Kneser solution of Eq. (1.1).

Proof of Theorem 1.4 According to conditions (1.19)-(1.21), there exist numbers $\delta_{0}>0$ and $\delta^{*}>\delta_{0}$ such that along with $(2.15)$ the inequality

$$
\varphi\left(v_{1}\left(\cdot ; \delta_{0}\right)\right)<c
$$

holds.

Let $w_{0}$ and $w_{1}$ be the functions given by equalities (2.7) and (2.14). Then, by virtue of (1.16), we have

$$
\lim _{b \rightarrow+\infty} w_{1}\left(t ; \delta_{0}, 0, b\right)=v_{1}\left(t ; \delta_{0}\right)
$$

uniformly on $[0, a]$. Thus from inequality (3.5) it follows the existence of a number $b_{0}>a$ such that

$$
\varphi\left(w_{1}\left(\cdot ; \delta_{0}, 0, b\right)\right)<c \text { for } b>b_{0} .
$$

Let $\left(b_{k}\right)_{k=1}^{+\infty}$ be a sequence of positive numbers such that

$$
b_{k} \geq b_{0}+k \quad(k=1,2, \ldots) .
$$


Then due to condition (3.6), there exists a sequence of positive numbers $\left(\varepsilon_{k}\right)_{k=1}^{+\infty}$ satisfying (2.37) and the inequalities

$$
\varphi\left(w_{1}\left(\cdot ; \delta_{0}, \varepsilon_{k}, b_{k}\right)\right)<c \quad(k=1,2, \ldots) .
$$

By Lemma 2.4 and conditions (2.15), (3.7), for any natural $k$, problem (1.1), (1.2) has a Kneser solution $u_{k}$ in the interval $\left[0, b_{k}\right]$ such that

$$
\delta_{0} \leq \delta_{k} \leq \delta^{*}, \quad u\left(b_{k}\right)=-\varepsilon_{k},
$$

where $\delta_{k}=u\left(b_{k}\right)$.

Without loss of generality, we can assume that the sequence $\left(\delta_{k}\right)_{k=1}^{+\infty}$ is converging. Put

$$
\delta=\lim _{k \rightarrow+\infty} \delta_{k}
$$

By Lemma 2.6, the sequence $\left(u_{k}\right)_{k=1}^{+\infty}$ contains a uniformly converging on every finite interval from $R_{+}$subsequence $\left(u_{k_{m}}\right)_{m=1}^{+\infty}$ such that the function, defined by the equality

$$
u(t)=\lim _{m \rightarrow+\infty} u_{k_{m}}(t) \quad \text { for } t \geq 0
$$

is a Kneser solution of problem (1.1), (1.14). On the other hand, if in the equality $\varphi\left(u_{k_{m}}\right)=c$ we pass to the limit as $m \rightarrow+\infty$, then it becomes clear that $u$ satisfies condition (1.2) as well. Thus $u$ is a Kneser solution of problem (1.1), (1.2).

To convince ourselves that Corollary 1.3 is valid, it suffices to note that if conditions (1.22)-(1.24) are fulfilled, then each of conditions (1.11), (1.16), (1.17) is satisfied iff $\alpha<2+\mu$ and $\beta>2+\mu$.

\section{Competing interests}

The authors declare that they have no competing interests.

\section{Authors' contributions}

All authors read and approved the final manuscript.

\section{Author details}

${ }^{1}$ A. Razmadze Mathematical Institute of I. Javakhishvili Tbilisi State University, 6 Tamarashvili St., Tbilisi, 0177, Georgia. ${ }^{2}$ International Black Sea University, 2 David Agmashenebeli Alley 13km, Tbilisi, 0131, Georgia. ${ }^{3}$ Faculty of Business and Management, Brno University of Technology, Kolejní 2906/4, Brno, 612 00, Czech Republic.

\section{Acknowledgements}

For the first author this work is supported by the Shota Rustaveli National Science Foundation (Project \# FR/317/5-101/12), and for the second author this work is supported by the Internal Grant Agency at Brno University of Technology (Project \# FP-S-13-2148).

Received: 11 February 2014 Accepted: 29 May 2014 Published online: 23 September 2014

\section{References}

1. Kneser, A: Untersuchung und asymptotische darstellung der integrale gewisser differentialgleichungen bei grossen reellen werthen der arguments, I. J. Reine Angew. Math. 116, 173-212 (1896)

2. Fermi, E: Un metodo statistico per la determinazione di alcune proprietà dell'atomo. Rend. R. Accad. Naz. Lincei 6 , 602-607 (1927)

3. Thomas, LH: The calculation of atomic fields. Proc. Camb. Philos. Soc. 23, 542-548 (1927)

4. Sansone, G: Ordinary Differential Equations, vol. I. Izdat. Inostrannol Literatury, Moscow (1953) (trans. in Russian)

5. Hartman, P, Wintner, A: On the non-increasing solutions of $y^{\prime \prime}=f\left(x, y, y^{\prime}\right)$. Am. J. Math. 73(2), 390-404 (1951)

6. Kiguradze, IT: On non-negative non-increasing solutions of non-linear second order differential equations. Ann. Mat. Pura Appl. 81, 169-192 (1969) 
7. Kiguradze, IT, Shekhter, BL: Singular boundary value problems for second order ordinary differential equations. Itogi Nauki Tekh., Ser. Sovrem. Probl. Mat., Novejshie Dostizh. 30, 105-201 (1987) (Russian). English transl.: J. Sov. Math. 43(2), 2340-2417 (1988)

8. Kiguradze, IT: On monotone solutions of nonlinear $n$-th order ordinary differential equations. Izv. Akad. Nauk SSSR, Ser. Mat. 33(6), 1373-1398 (1969) (Russian). English transl.: Math. USSR, Izv. 3, 1293-1317 (1969)

9. Kiguradze, IT: Some Singular Boundary Value Problems for Ordinary Differential Equations. Tbilisi University Press, Tbilisi (1975) (Russian)

10. Kiguradze, IT, Rachůnková, I: On the solvability of a nonlinear Kneser type problem. Differ. Uravn. (Minsk) 15(10), 1754-1765 (1979) (Russian). English transl.: Differ. Equ. 15, 1248-1256 (1980)

11. Chanturia, TA: On the Kneser type problem for systems of ordinary differential equations. Mat. Zametki 15(6), 897-906 (1974) (Russian)

12. Coffman, CV: Non-linear differential equations on cones in Banach spaces. Pac. J. Math. 14(1), 9-15 (1964)

13. Hartman, P, Wintner, A: On monotone solutions of systems of non-linear differential equations. Am. J. Math. 76(4), 860-866 (1954)

14. Kiguradze, IT, Rachůnková, I: On a certain nonlinear problem for two-dimensional differential systems. Arch. Math. 15(1), 15-38 (1980)

15. Rachůnková, I: On the Kneser problem for a system of nonlinear ordinary differential equations. Soob. Acad. Nauk GSSR 94(3), 545-548 (1979) (Russian)

16. Rachůnková, I: On a Kneser problem for a system of nonlinear ordinary differential equations. Czechoslov. Math. J. 31(1), 114-126 (1981)

17. Kiguradze, I: On Kneser solutions of the Emden-Fowler differential equation with a negative exponent. Tr. Inst. Mat. NAN Belarusi 4, 69-77 (2000)

doi:10.1186/s13661-014-0147-x

Cite this article as: Partsvania and Pưža: The nonlinear Kneser problem for singular in phase variables second-order differential equations. Boundary Value Problems 2014 2014:147.

\section{Submit your manuscript to a SpringerOpen ${ }^{\circ}$ journal and benefit from:}

- Convenient online submission

- Rigorous peer review

- Immediate publication on acceptance

- Open access: articles freely available online

- High visibility within the field

- Retaining the copyright to your article 